\title{
On Ultrasonic Communication through Metal Structure for Machine Embedded Sensing
}

\author{
Jinjiang Wang ${ }^{1,}$, Xinyao Tang ${ }^{2}$, Robert X. Gao ${ }^{2}$, Lixiang Duan ${ }^{1}$, and Laibin Zhang ${ }^{1}$ \\ ${ }^{1}$ School of Mechanical and Transportation Engineering \\ China University of Petroleum, Beijing, China, 102249 \\ ${ }^{2}$ Department of Mechanical Engineering \\ University of Connecticut, Storrs, CT 06269, USA \\ * Corresponding author: jwang@ @up.edu.cn
}

\begin{abstract}
With the development of machine embedded sensing, wireless communication through metal structures is becoming a critical issue in manufacturing equipment, process monitoring and control. To address it, this paper presents a multi-carrier ultrasonic communication scheme to achieve high-bit data rate for machine embedded sensing through metal structures. To extract the coded information from ultrasonic signal and denoise the signal, a new signal processing method, named multi-scale enveloping symbolic analysis (MuSEnSA), is also formulated by integrating dual-tree complex wavelet packet transform and symbolic analysis. Dual-tree complex wavelet packet transform (DT-CWPT) is firstly investigated by separating multi-carrier signals under noise contamination, given its properties of shift-invariance and flexible time frequency partitioning. A new envelope extraction and threshold setting strategy for selected wavelet coefficients is then introduced to retrieve the coded digital information by taking advantage of symbolic analysis. Numerical and experimental studies are performed to evaluate the effectiveness of the developed signal processing method for injection molding monitoring.
\end{abstract}

Keywords: Ultrasonic communication, dual-tree complex wavelet packet transform, symbolic analysis, threshold setting strategy 


\section{INTRODUCTION}

Machinery condition monitoring is of critical importance to improving product quality, minimizing material waste and reducing operational cost in modern manufacturing systems, thus has attracted extensive attention. Data acquisition and transmission play important roles in machinery condition monitoring to enable data gathering and information sharing [1]. To acquire direct machinery status, in-situ machine-embedded sensors have been investigated with enhanced resolution and sensitivity for online process monitoring and control [2]. Such embedded sensors are commonly placed within the metal structures, which have a great impact on data transmission. Due to the electromagnetic shielding effect in the metal structures, commonly used wireless communication techniques [3] such as a radio frequency (RF) or infrared (IR) system are not applicable. Wired data transmission is also limited, since throughhole cables are usually hard to be configured in complex metal tooling structures. On the other hand, ultrasonic communication has recently attracted much attention to overcome electromagnetic shielding in wireless data transmission through metal structures [4].

Much effort has been reported on investigating ultrasonic communication techniques in different applications, such as monitoring of enclosed shipping container, embedded sensors for online process monitoring (e.g. primary shaping, metal forming and cutting). One typical ultrasonic communication system consists of two ultrasonic transducers including one transmitter and one receiver as shown in Fig. 1. The transmitter is usually located within the metal enclosure, while the receiver is located outside of the metal enclosure. The transmitter sends the ultrasonic waves as the carriers with different modulations to the receiver end. Three different modulation mechanisms including amplitude modulation, frequency modulation, and binary phase modulation are investigated in [5] for ultrasonic communications. The results show that binary phase modulation has the lowest bit error rate, while amplitude modulation is easy to implement 
and requires minimal hardware. In [6], an orthogonal frequency division multiplexing (OFDM) scheme is investigated to evaluate the frequency-selective fading effect in ultrasonic communications. A multi-tone frequency modulation scheme is also investigated in [7], and the transmitted digital symbols are obtained from the spectrum of the received ultrasonic waves through FFT analysis.

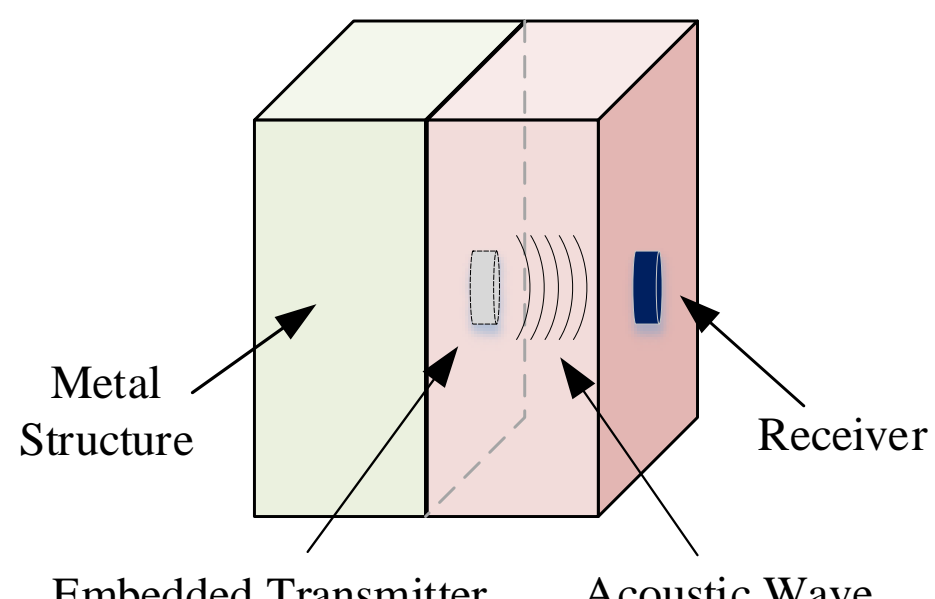

Fig. 1. A typical ultrasonic system for communication through a metal structure.

Ultrasonic communication provides a viable solution for data transmission through metal structures. However, the key challenges associated with the ultrasonic signal are inherent signal attenuation [8], wave diffraction and reflection in metal interfaces, which brings about significant issues such as inter-symbol inference and data loss in ultrasonic communications [9]. To address these challenges, various signal processing techniques, such as signal averaging, matched filtering, frequency spectrum analysis, autoregressive analysis, and the wavelet transform, have been utilized to analyse ultrasonic signals [10]. Among these signal processing techniques, the wavelet transform has been widely used for ultrasonic signal processing. In [11], a continuous Gaussian wavelet transform is investigated for ultrasonic pulse detection. The discrete wavelet transform and wavelet packet transform have been compared in [12] for signal-to-noise ratio enhancement, and the wavelet packet transform shows better performance. An adaptive Morlet 
wavelet filter based on the Morlet wavelet and least mean squares (LMS) filter is also investigated for estimating time-of-flight diffraction in ultrasonic signal analysis [13]. In [14], a signal matching wavelet (SMW) method is developed to optimize the matching between the ultrasonic signal and base wavelet.

As the above analysis shows, ultrasonic communication and the wavelet transform have been widely investigated. However, past work on ultrasonic communication is mainly focused on a single-carrier signal, which limits the data rate in transmission. It is also known that the conventional wavelet transform has a shift varying issue, which may cause errors in retrieving the digital symbols in ultrasonic communication. To address these issues and denoise the signal, this paper firstly investigates a multi-carrier coded-ultrasonic wave modulation [15] scheme to improve high-bit data rate in ultrasonic communication. A dual-tree complex wavelet packet transform [16] based approach is then presented to denoise the signal by decomposing the signal into a number of wavelet coefficients in different frequency bands. The dual-tree complex wavelet packet transform is selected because of its attractive properties of shift-invariance and flexible time-frequency partition property. A new envelope extraction and threshold setting strategy is then investigated to retrieve coded digital information. Numerical and experimental studies have been performed to validate the effectiveness of the presented method.

The rest of the paper is constructed as follows. The theoretical background of dual-tree complex wavelet packet transform (DT-CWPT) is firstly discussed in Section 2. The theoretical framework of the developed ultrasonic signal retrieval method is then presented in Section 3, including multi-carrier coded-acoustic modulation scheme, DT-CWPT based multi-resolution signal separation, envelope extraction and threshold setting strategy. Numerical simulations are performed to investigate the performance of developed method under the noise contamination. 
Next the effectiveness of the developed method is demonstrated in the experimental studies on a typical injection molding machine in Section 4. The conclusions are finally drawn in Section 5.

\section{DUAL-TREE COMPLEX WAVELET PACKET TRANSFORM}

The wavelet transform is a multi-resolution analysis technique which can be used for timefrequency representation of signals. It is achieved by scaling and shifting operations on the base wavelet $\psi(t)$, where the wavelet transform of a signal $x(t)$ with finite energy can be obtained by:

$$
w t(s, \tau)=|s|^{-1 / 2} \int_{-\infty}^{\infty} x(t) \bar{\psi}\left(\frac{t-\tau}{s}\right) d t(1)
$$

where the symbol $s$ represents the scaling parameter, which dilates or contracts the base wavelet, and $\tau$ is the shifting parameter that translates the wavelets along the time axis. $\bar{\psi}(\bullet)$ represents the complex conjugate of the scaled and shifted base wavelet. The discrete wavelet transform has been widely used for signal processing since it has high computational efficiency and easy invertibility, and its structure is shown in Fig. 2. The input signal is first divided into two wavelet sub-bands by a filter bank using high pass and low pass filters, then the lower frequency subband is decomposed by high pass and low pass filters as shown in Fig. 2a, in which $G(\omega)$ and $H(\omega)$ represent the high pass and low pass filters, respectively. These filters are implemented through scaling the wavelet function. However, this fixed frequency partition manner causes low frequency resolution at high wavelet sub-bands. The wavelet packet transform is then introduced to enhance the frequency resolution in high frequency sub-bands. The decomposition is performed on both frequency sub-bands as shown in Fig. $2 b$, thus has more flexibility in frequency partition manner. 


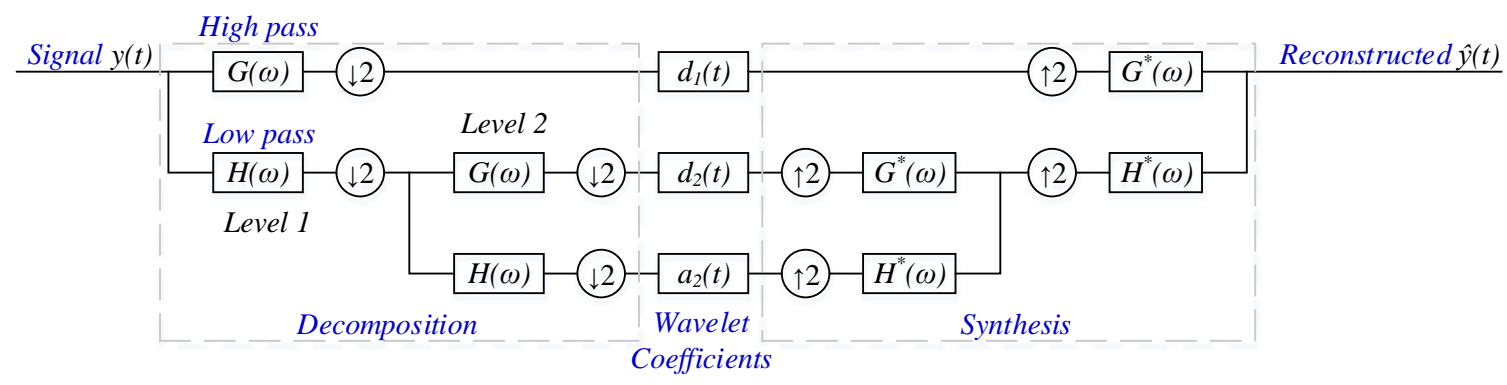

(a) Two level decomposition structure in discrete wavelet transform

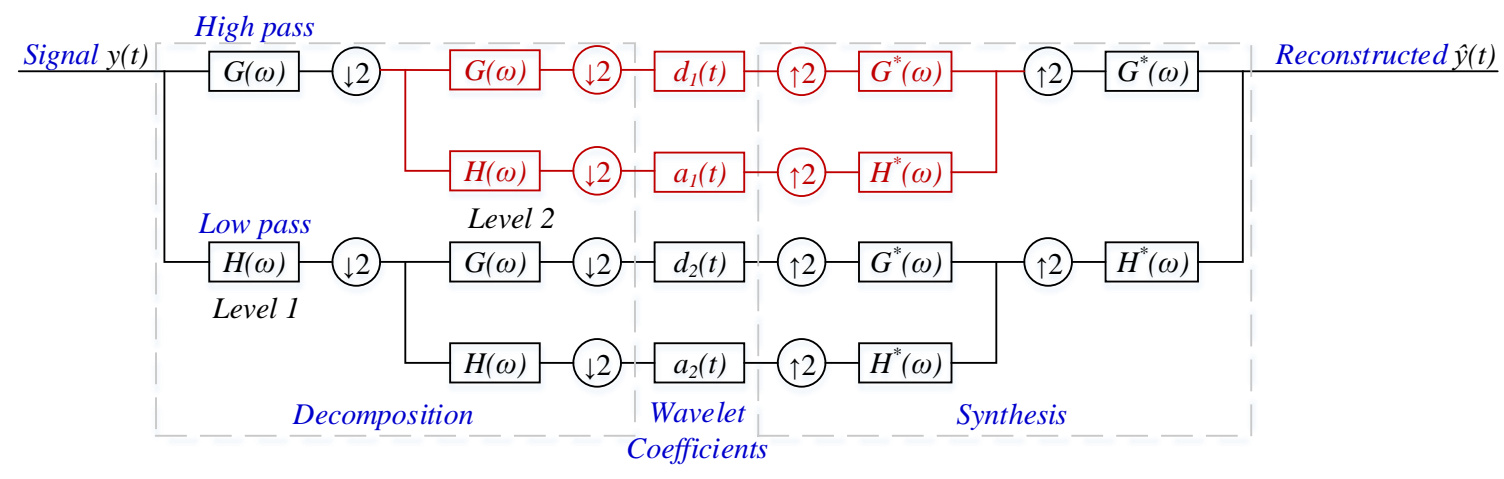

(b) Two level decomposition structure in wavelet packet transform

Fig. 2. Comparison between discrete wavelet transform and wavelet packet transform.

The wavelet transform has proved to be a powerful tool for signal processing. However, the wavelet transform commonly uses the real oscillating base wavelet, which leads to non-smooth wavelet coefficients and causes the shift variance issue. Fig. 3a shows the decomposed four-level wavelet coefficients obtained by discrete wavelet transform on a set of shifted step functions $\{\delta(t-i), i=1,2, \ldots, 16\}$. From the results, wavelet coefficients at different levels present obvious disorder and shift variance. One approach to address these issues is the dual-tree complex wavelet transform (DT-CWT), which was introduced by Kingsbury [17]. In addition to one wavelet filter bank in discrete wavelet transform, it introduces another wavelet filter bank according to a certain criterion. The first wavelet filter bank is the real part, while the second wavelet filter bank is the imaginary part. The impulse responses of second wavelet filter are approximately the discrete Hilbert transforms of those of the first wavelet filter bank. Thus the frequency responses of the dual-tree complex wavelet transform (DT-CWT) are approximately 
analytical, thus the decomposed wavelet coefficients present the orderly translation and near shift variance as shown in Fig. $3 b$.

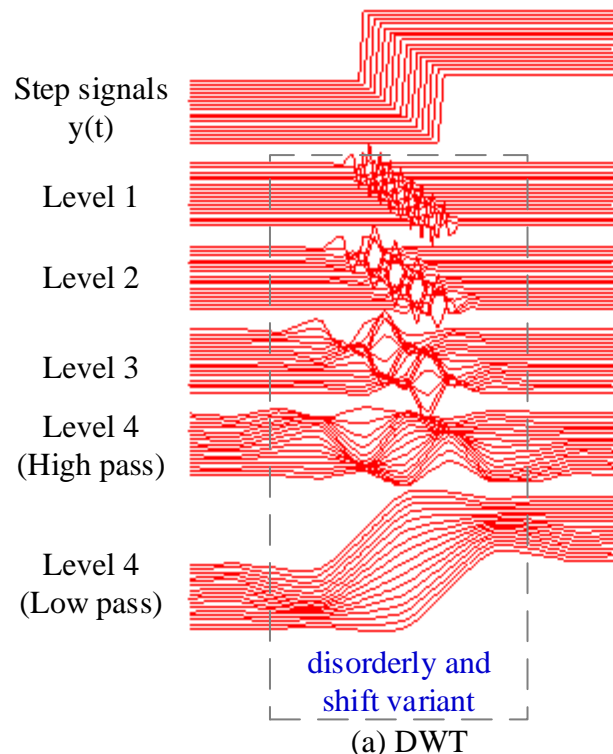

(a) DWT

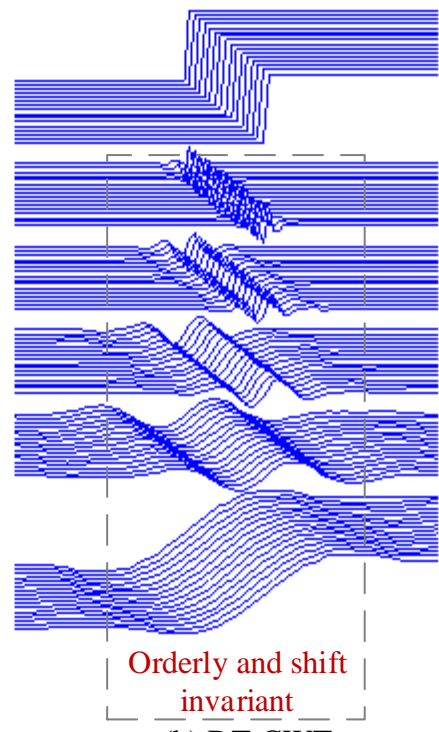

(b) $\overline{\mathrm{DT}} \overline{\mathrm{C}} \overline{\mathrm{CWT}}$

Fig. 3. Performance comparison between DWT and DT-CWT on a series of step signals.

Following the idea of DT-CWT, the dual-tree complex wavelet packet transform was firstly formulated in $[16,18]$. The challenge is constructing two wavelet filter banks being analytic. Based on the proof in [19], they need to satisfy the half sample delay condition and top level condition. Top level condition requires that the filters in the first decomposition level are different from the subsequent decomposition levels. At the first level, the imaginary-part filter is one sample delayed version of the corresponding real-part filter.

$$
\begin{aligned}
& G_{(1)}^{\mathrm{Im}}(n)=G_{(1)}^{\mathrm{Re}}(\mathrm{n}-1) \\
& H_{(1)}^{\mathrm{Im}}(n)=H_{(1)}^{\mathrm{Re}}(\mathrm{n}-1)
\end{aligned}
$$

where $G_{(1)}^{\mathrm{Im}}(\mathrm{n})$ and $H_{(1)}^{\mathrm{Im}}(\mathrm{n})$ denote the imaginary-part high pass and low pass filters, and $G_{(1)}^{\mathrm{Re}}(\mathrm{n})$ and $H_{(1)}^{\mathrm{Re}}(\mathrm{n})$ represent the real-part high pass and low pass filters at the first decomposition level, respectively. The subsequent decomposition levels meet the half sample delay condition which 
requires that imaginary part of the filter is a half sample shifted version of the corresponding real part filter.

$$
\begin{aligned}
& G^{\operatorname{Im}}(n)=G^{\mathrm{Re}}(\mathrm{n}-0.5) \\
& H^{\operatorname{Im}}(n)=H^{\mathrm{Re}}(\mathrm{n}-0.5)
\end{aligned}
$$

where $G^{\mathrm{Im}}(\mathrm{n})$ and $H^{\mathrm{Im}}(\mathrm{n})$ denote the imaginary part filters while $G^{\mathrm{Re}}(\mathrm{n})$ and $H^{\mathrm{Re}}(\mathrm{n})$ represent the real part filters at the subsequent decomposition levels. The first wavelet $\varphi(t)$ is defined by [16]:

$$
\varphi(\mathrm{t})=\sqrt{2} \sum_{n} G^{\mathrm{Re}}(\mathrm{n}) \phi(2 \mathrm{t}-\mathrm{n})
$$

where $\phi(\mathrm{t})$ is formulated as:

$$
\phi(\mathrm{t})=\sqrt{2} \sum_{n} H^{\mathrm{Re}}(\mathrm{n}) \phi(2 \mathrm{t}-\mathrm{n})
$$

The wavelet associated with the second wavelet filter bank is denoted as $\varphi^{\prime}(\mathrm{t})$. It is the Hilbert transform of the first wavelet $\varphi(t)$.

$$
\varphi^{\prime}(\mathrm{t})=\mathbf{H}\{\varphi(\mathrm{t})\}
$$

where $\mathbf{H}\{\cdot\}$ represents the Hilbert transform. According to the property of Hilbert transform, the low pass filter in the second wavelet filter bank $\left\{G^{\operatorname{Im}}(\mathrm{n}), H^{\operatorname{Im}}(\mathrm{n})\right\}$ is equal to the half-sampled delayed version of first wavelet filter bank $\left\{G^{\mathrm{Re}}(\mathrm{n}), H^{\mathrm{Re}}(\mathrm{n})\right\}$. Therefore, the half sample delay condition is satisfied. In the frequency domain, the two wavelets can be derived as [16]:

$$
\begin{gathered}
H^{\operatorname{Im}}\left(e^{j \omega}\right)=e^{-j 0.5 \omega} H^{\operatorname{Re}}\left(\mathrm{e}^{\mathrm{j} \omega}\right) \\
\mathrm{G}^{\operatorname{Im}}\left(e^{j \omega}\right)=-j \operatorname{sgn}(\omega) e^{j 0.5 \omega} \mathrm{G}^{\mathrm{Re}}\left(\mathrm{e}^{\mathrm{j} \omega}\right)
\end{gathered}
$$

where $\operatorname{sgn}(\cdot)$ is the signum function. Then, for an input signal $y(t)$, two wavelet filter banks operate in parallel. Both the high pass and low pass filters follow the wavelet coefficients in the 
previous stage. Following the procedure, the structure of dual-tree complex wavelet packet transform is illustrated in Fig. 4. Due to the analytic property in dual-tree complex wavelet packet transform, the envelope of the signal can be readily calculated from the modulus of the wavelet coefficients.

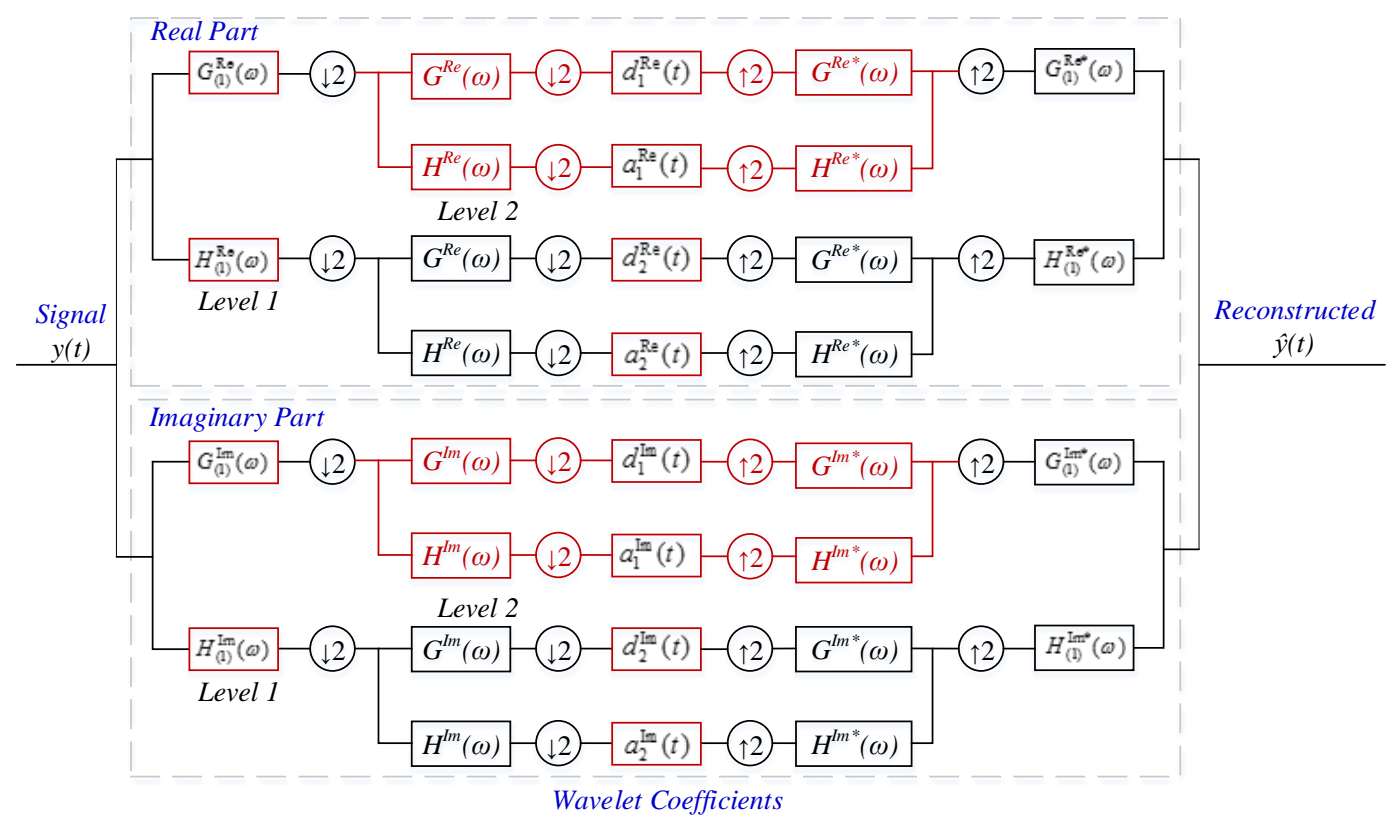

Fig. 4. A two-level decomposition structure in dual-tree complex wavelet packet transform implemented using two filter banks.

Based on the above analysis, it can be concluded that dual-tree complex wavelet packet transform has the following benefits that are essential in ultrasonic signal processing:

1) Flexible frequency partition manner;

2) Shift invariance;

3) Signal separation and envelope extraction in one step.

\section{FORMULATION OF ULTRASONIC SIGNAL RETRIEVAL METHOD}

Data communication through metal structures requires a form of modulation, involving the change in amplitude, frequency, or phase of the carrier signal according to the transmitted data. 
There are in essence three techniques: amplitude modulation, frequency modulation, and phase modulation. An amplitude modulation scheme is selected in this paper, since it is easy to be implemented in embedded sensors with minimum hardware requirements. To improve the data transmission rate, a multi-carrier amplitude modulation scheme is presented on the transmitter side. It introduces significant inter-symbol inference and data loss due to inherent signal attenuation, wave diffraction and reflection in the metal structure. A multi-scale enveloping symbolic analysis (MuSEnSA) method based on DT-CWPT and symbolic representation analysis is proposed for signal processing in order to recover data from the ultrasonic carrier signals, the details of which are described below.

\subsection{Multi-carrier amplitude modulation scheme}

To transmit the sensing measurement of an embedded sensor within the metal structure to a receiver placed outside of the metal structure, ultrasonic waves have been recently investigated as the information carriers $[4,6,7]$. In the amplitude modulation scheme (also known as on-off keying modulation), the sensing measurement is converted into binary digital form consisting of ones and zeros. The binary digital data is then modulated with carrier waves, in which zeros represent no carrier wave, while ones denote the carrier wave. The sequence of the amplitudes of a carrier wave stands for the sensing measurement to be transmitted. In the conventional amplitude modulation scheme, a single carrier is usually used, which means the data needs to be transmitted sequentially. To improve the data transmission rate, a multi-carrier amplitude modulation scheme is presented in this paper as shown in Fig. 5. Multiple carrier waves with different frequencies (e.g., $f_{1}$ and $f_{2}$ ) are strategically selected based on the property of the ultrasonic transmitter. Multiple parameters (e.g., temperature, pressure, etc.) in the sensing measurement are then digitized and modulated with carrier waves, respectively. The modulated 
carrier waves are then mixed using the $S U M$ operation, and the mixed signal is sent to the ultrasonic transmitter. Therefore, in a multi-carrier amplitude modulation scheme multiple parameters can be transmitted in parrallel to improve the data transmission rate.

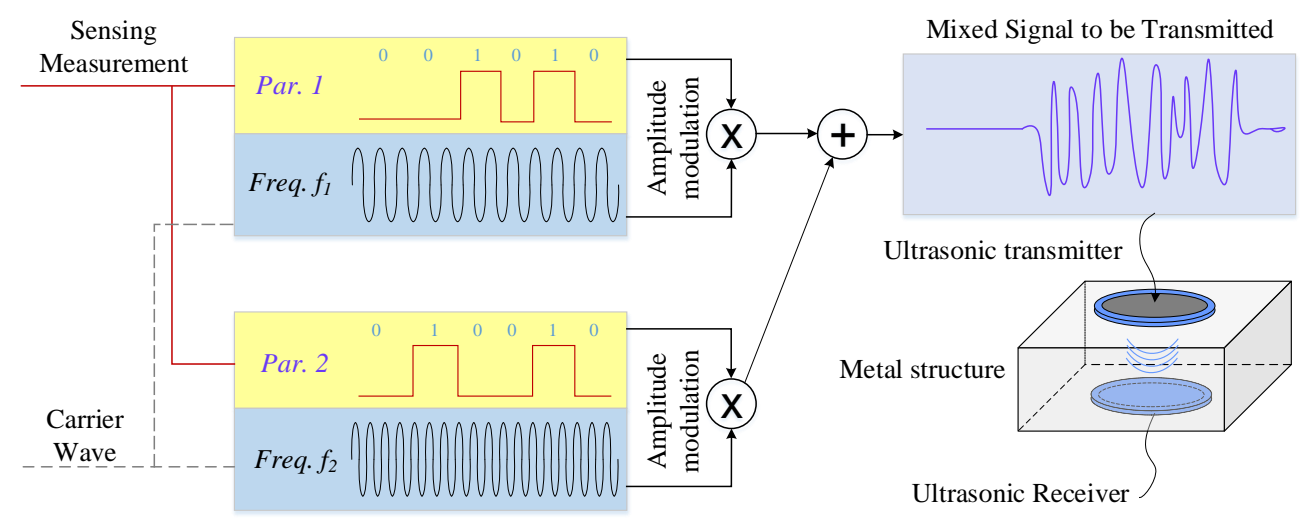

Fig. 5. Multi-carrier amplitude modulation scheme.

\subsection{DT-CWPT based ultrasonic signal retrieval}

Due to inherent signal attenuation, wave diffraction, and multi-path effect in the metal structure, significant inter-symbol affects the ultrasonic signal. DT-CWPT is investigated to denoise the signal and retrieve the sensing measurement as shown in Fig. 6. It has attractive properties including flexible frequency partition property, shift invariance, and the integration of signal separation and envelope extraction in one step as discussed in Section 2.

The noisy signal obtained by the ultrasonic receiver is firstly decomposed by DT-CWPT into different levels of wavelet coefficients to separate the ultrasonic waves in the timefrequency domain. Next, the representative wavelet coefficients corresponding to the ultrasonic carrier waves are then selected based on the maximum relative energy to Renyi entropy ratio criteria [20]. The envelopes of wavelet coefficients are then extracted to demodulate the coded sensing measurement from the carrier waves. Symbolic analysis is then used to retrieve the digitized data from the envelopes of wavelet coefficients. 


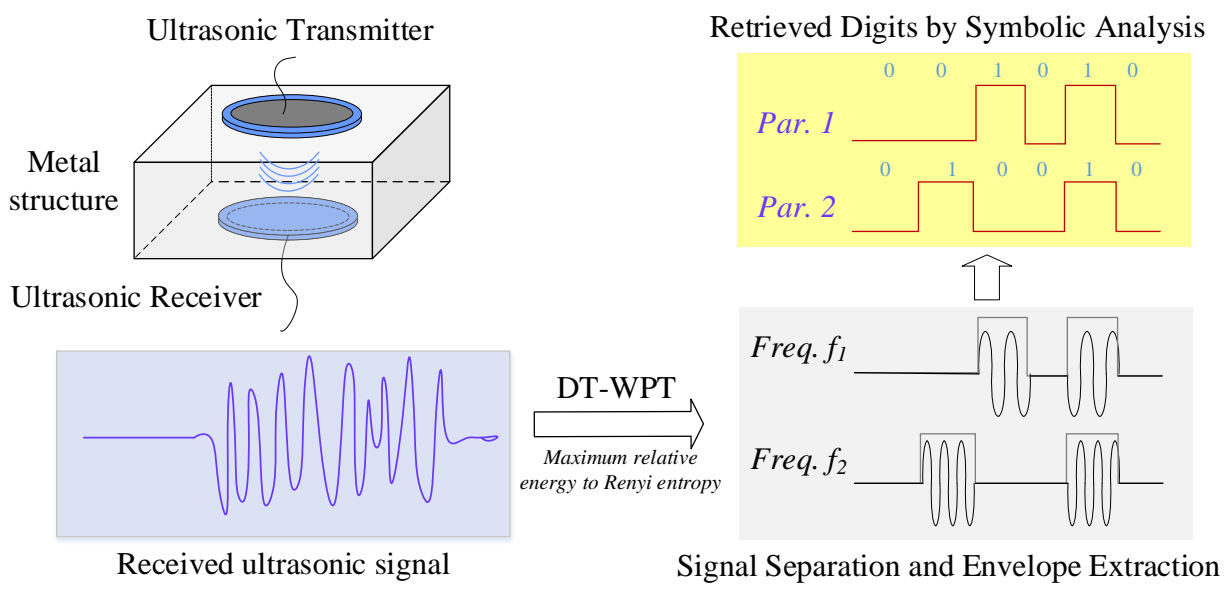

Fig. 6. Ultrasonic signal demodulation and symbol retrieval.

\subsection{Maximum relative energy to Renyi entropy ratio criteria}

It is important to select the representative wavelet coefficients from DT-CWPT decomposition results for further analysis. The energies of acoustic carrier waves locate in the narrow bands corresponding to particular scales/levels in the DT-CWPT coefficients. The scale/level which has the relative high amount of energy becomes the best-suited one. On the other hand, quadratic Renyi entropy can be used to measure the variation of spectral distribution. The scale/level corresponding to the acoustic carrier wave has the lower entropy, while the one corresponding to noise has the higher entropy. Based on the properties of the decomposed wavelet coefficients of the acoustic wave, maximum relative energy to Renyi entropy ratio is investigated. Generally, the energy associated with the wavelet coefficient at each scale is calculated as:

$$
E(s)=\sum_{\tau=1}^{m}|w t(s, \tau)|^{2}
$$

where $m$ is the length of wavelet coefficients, and $w t(s, \tau)$ represents the DT-CWPT coefficient at scale/level $s$. The total energy of a signal after wavelet transform is given by:

$$
E_{\text {total }}=\sum_{s} \sum_{\tau}|w t(s, \tau)|^{2}=\sum_{s} E(s)
$$


A normalized value relative wavelet energy can be defined as:

$$
p_{s}=\frac{E(s)}{E_{\text {total }}} ; \text { where } \sum_{s} p_{s}=1
$$

In Eq. (13), $p_{s}$ represents the energy probability distribution across scales. The scale/level with the higher relative wavelet energy can be used as a measure for scale/level selection.

To select the optimal scale/level where the frequencies of acoustic carrier waves are dominant, Quadratic Renyi entropy [21] has been investigated to measure the variation of spectral distribution of wavelet coefficients. The spectral probability distribution of DT-CWPT coefficients at scale/level $s$ is defined as:

$$
p_{s, f}=\frac{|w t(s, f)|^{2}}{\sum_{f}|w t(s, f)|^{2}} ; \text { where } \sum_{f} p_{s, f}=1
$$

here, $w t(s, f)$ is the Fourier transform of $w t(s, \tau)$. Accordingly, Quadratic Renyi entropy associated with the wavelet spectrum at scale/level $s$ can be obtained as:

$$
H_{2}(s)=-\log _{2}\left(\sum_{f} p_{s, f}^{2}\right)
$$

where the scale/level with the minimum Renyi entropy is selected as the optimal scale. Such a scale is characterized by the maximum relative energy (corresponding to the structural resonant frequency) and minimum Renyi entropy of the DT-CWPT coefficients, and is chosen as the bestsuited scale/level for acoustic carrier waves. Accordingly, a scale selection criterion called maximum relative energy to Renyi entropy ratio is formulated as:

$$
\rho(s)=\frac{p_{s}}{H_{2}(s)}
$$

\subsection{Symbolic representation analysis}

The envelope demodulated from an ultrasonic carrier wave by DT-CWPT is still time series data. To retrieve the digital binary data from the envelope of the wavelet coefficients, symbolic 
representation analysis is investigated [22]. Symbolic representation analysis is to divide a signal into equal-sized sections followed by comparing each section with a threshold. More specifically, symbolic representation analysis takes a real valued signal and divides it into equal sized sections. The length of each section is equal to the pre-set time window in the amplitude modulation scheme. The mean value of each section is then calculated, and compared with the threshold. Considering acoustic signal attenuation in the metal structure, the threshold is adaptively set based on the transmitted acoustic signal. The ultrasonic transmitter initially transmits one digitized data such as 0x55, 0xAA in a hexadecimal form by modulating with the carrier waves. The threshold is determined by distinguishing the 0's and 1's in the received signal as shown in Fig. 7.

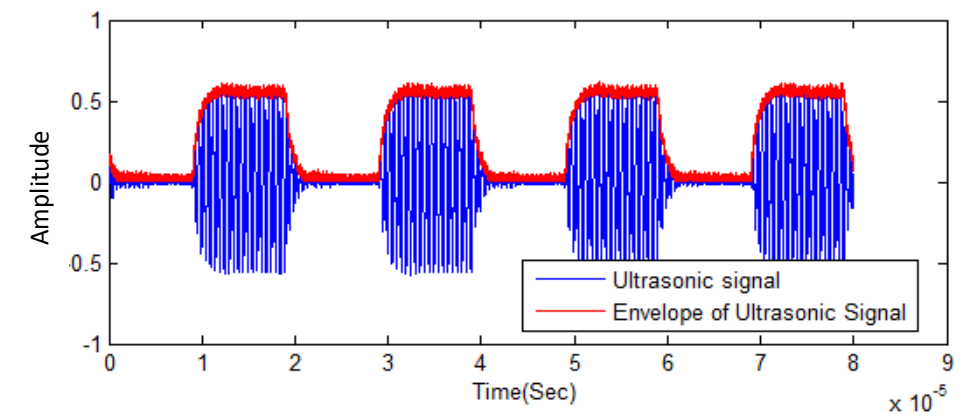

(a) Ultrasonic signal and its envelope

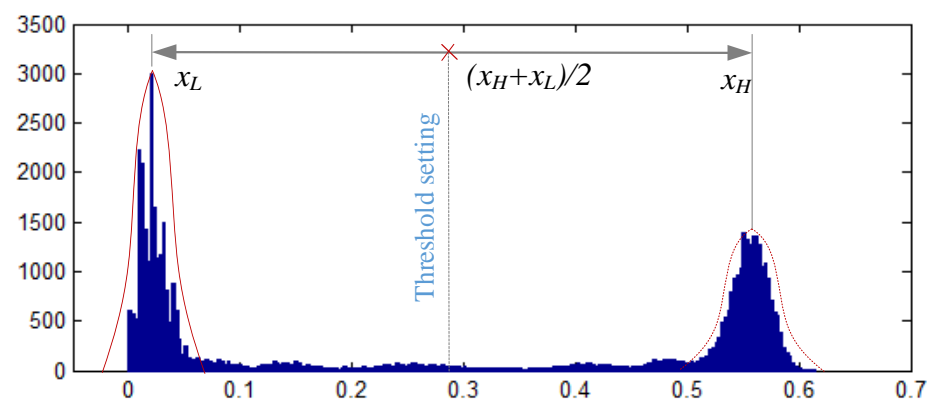

(b) Histogram of the envelope of ultrasonic signal

Fig. 7. Illustration of the threshold setting in symbolic representation analysis.

The received ultrasonic signal and its envelope are shown in Fig. 7a, and the histogram of the envelope of the ultrasonic signal is then illustrated in Fig. 7b. From the two peaks at $x_{L}$ and 
$x_{H}$ corresponding to the binary values 0 's and 1 's, the threshold can be determined as $\left(x_{L}+x_{H}\right) / 2$. By comparing the time series data to the threshold, binary digits (e.g., 0's and 1's) are produced from the envelope of the wavelet coefficients.

\subsection{Numerical Simulation}

Amplitude modulation is easily implemented, where the symbols are represented as discrete amplitudes of a fixed frequency carrier. In the binary amplitude modulation, where only two symbol states are needed, the carrier is simply turned on or off. In the presented multi-carrier amplitude modulation scheme, multiple carriers with different frequencies are used. By taking two carriers as an example, the general form for a multi-carrier amplitude modulation signal $s(t)$ can be written mathematically as:

$$
s(t)=a(t) \sin \left(2 \pi f_{a} t+\varphi_{a}\right)+b(t) \sin \left(2 \pi f_{b} t+\varphi_{b}\right)+\text { noise }(t)
$$

where $a(t)$ is the amplitude of the first carrier with the frequency $f_{a}$, and $b(t)$ is the amplitude of the second carrier with the frequency $f_{b} . \varphi_{a}$ and $\varphi_{b}$ are the phases of two carriers, respectively. By setting $f_{a}$ as $800 \mathrm{kHz}, f_{b}$ as $1 \mathrm{MHz}, a(t)$ as the sequence of $(01011100), b(t)$ as the sequence of (10001111), and $\varphi_{a}$ and $\varphi_{b}$ as zero degree, the simulated ultrasonic is obtained as shown in Fig. 8.

Here white noise is added with signal-to-noise ratio (SNR) of $10 \mathrm{~dB}$ to simulate the experimental conditions. The spectrum analysis of the ultrasonic signal is shown in Fig. $8 \mathrm{~b}$, in which the carrier frequencies of $800 \mathrm{kHz}$ and $1 \mathrm{MHz}$ are clearly identified. Next, DT-CWPT with the decomposition level 4 is performed on the simulated ultrasonic signal, and the decomposition results of DT-CWPT are obtained as shown in Fig. 9. The real parts of wavelet coefficients are highlighted in blue colour, while the imaginary parts are labelled in red. According to the criteria maximum relative energy to Renyi entropy ratio, two wavelet 
coefficients are selected and highlighted in dashed blue colours corresponding to two ultrasonic carrier waves at the frequencies of $0.8 \mathrm{MHz}$ and $1 \mathrm{MHz}$, respectively.

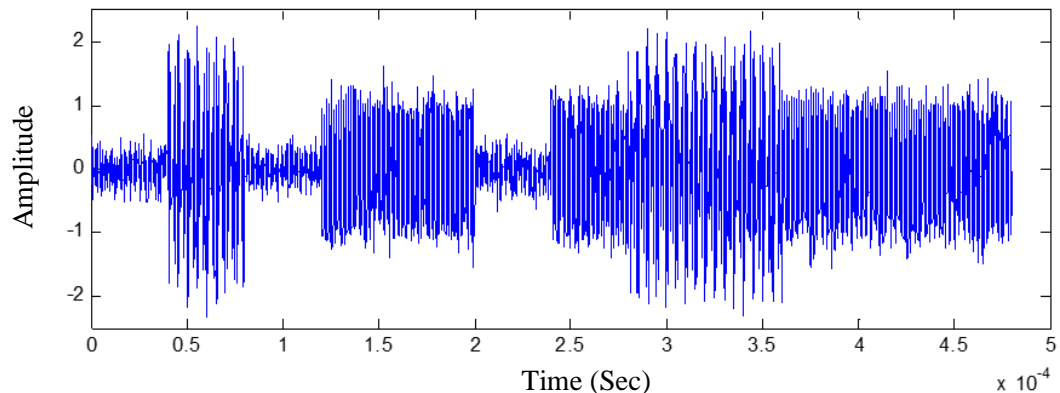

(a)

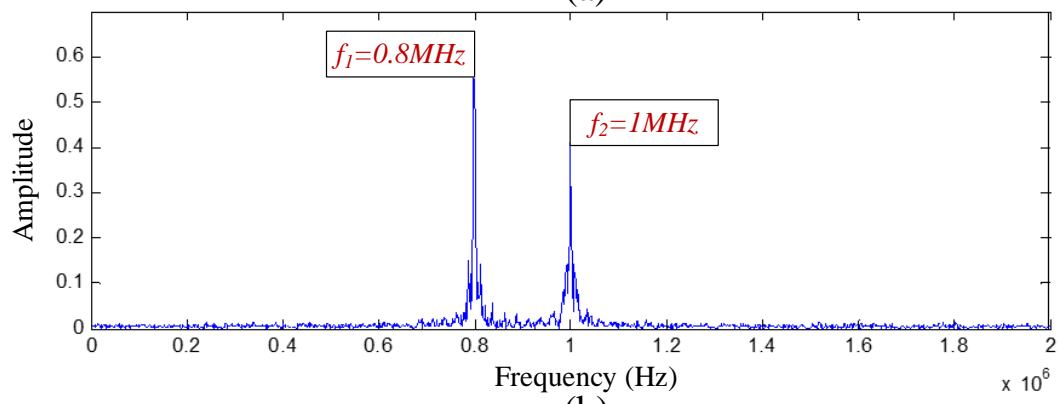

(b)

Fig. 8. Simulated ultrasonic signal and its spectrum.
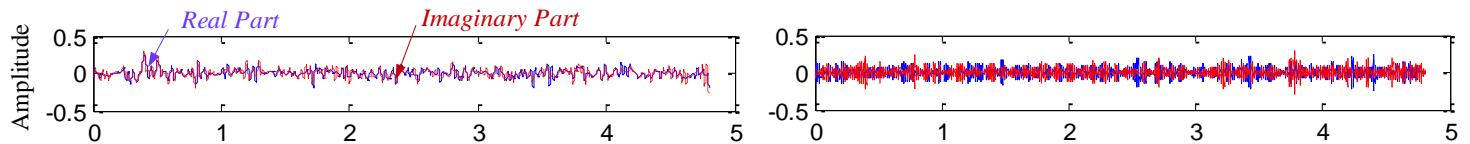

$\times 10^{-4}$
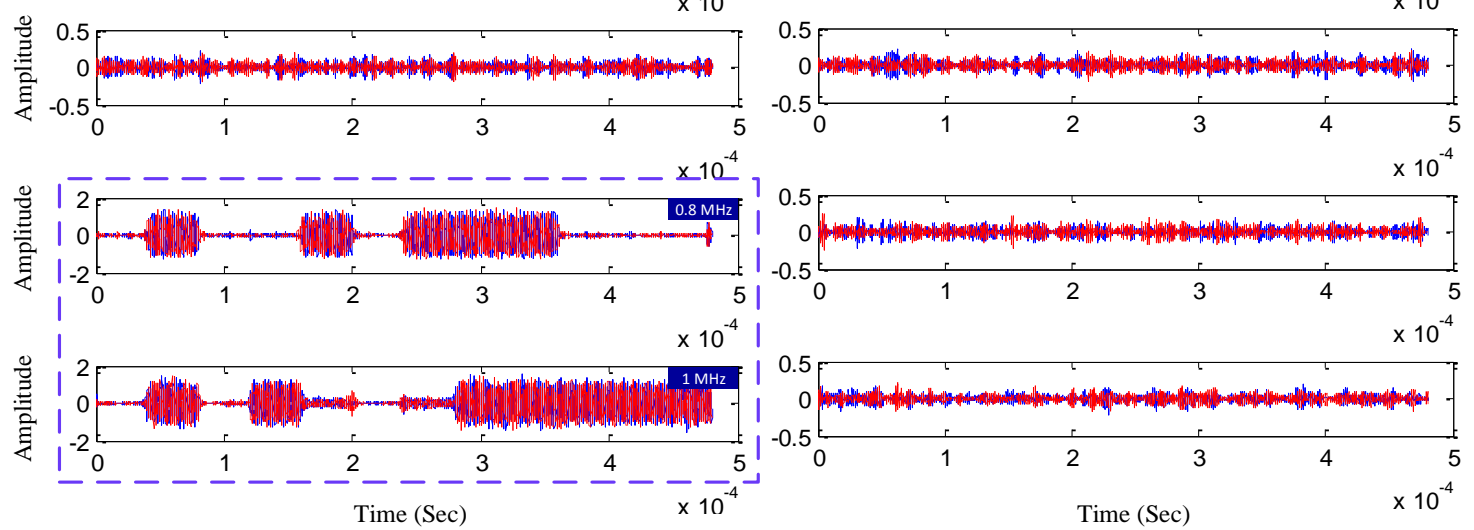

Fig. 9. Part of DT-CWPT decomposition results of simulated ultrasonic signal at the decomposition level 4 (real part and imaginary part). 
According to the criterion of DT-CWPT, the envelopes of the wavelet coefficients are extracted by the Absolute value operation of real part and imaginary part as shown in Fig. 10. By identifying the head (bits 10) of the data, the envelopes are then divided into preset-sized sections. The mean value of each section is calculated, and then compared with the threshold as discussed in Section 3.4. The binary symbols (e.g., 0 or 1) can be retrieved from the ultrasonic signal. As illustrated in Fig. 10, the 8-bit digital symbols (01011100) and (10001111) are clearly retrieved. The parity bit after the 8-bit digital symbols is used to check the symbol error rate.

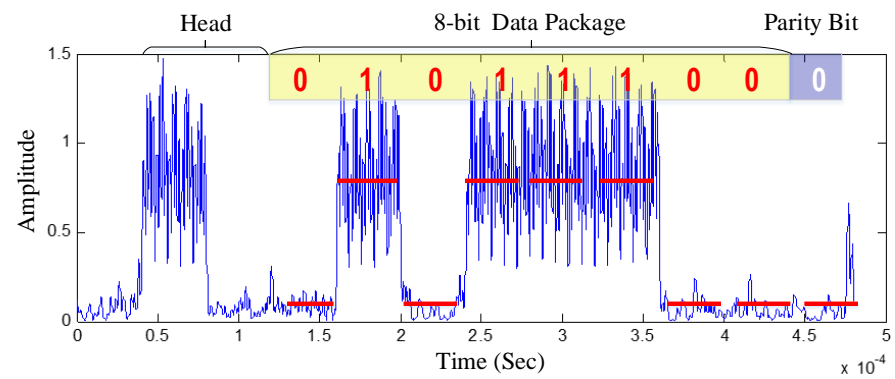

(a)

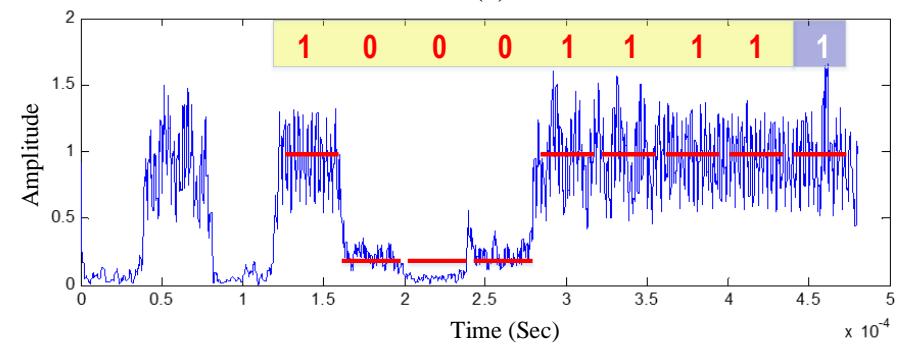

(b)

Fig. 10. The results of the extraction operation of the wavelet coefficients of simulated ultrasonic signal and retrieved digital data using symbolic representation analysis with carrier frequency, a) $0.8 \mathrm{MHz}$, and b) $1 \mathrm{MHz}$.

\section{EXPERIMENTAL STUDIES}

Over the past decades, machine-embedded sensors that can simultaneously measure two or more process parameters and wirelessly transmit data have gained increasing interest from the industry and research community. To evaluate the DT-CWPT based ultrasonic signal retrieval method, a representative injection molding machine (model SD750UZ) as shown in Fig. 11 is 
utilized, where the parameters temperature and pressure in the cavity are important for condition monitoring and quality control in the injection molding machine. Due to the complex tooling paths in injection mold, it is usually undesirable to put a through-hole to install the sensors for wired signal transmission. Thus wireless communication is preferred for machinery embedded sensors. A custom-designed wireless multivariate sensor (MVS) was structurally embedded within the mold cavity [15], as shown in Fig. 11. An ultrasonic receiver is placed outside of the $40 \mathrm{~mm}$-thick mold plate for signal reception. To evaluate the performance and robustness of the acoustic-based wireless data transmission method, the digitized signals carrying measured information (e.g., melt temperature and melt pressure) are recorded in the MVS and then compared with the signal received by an ultrasound receiver placed outside of the mold cavity.
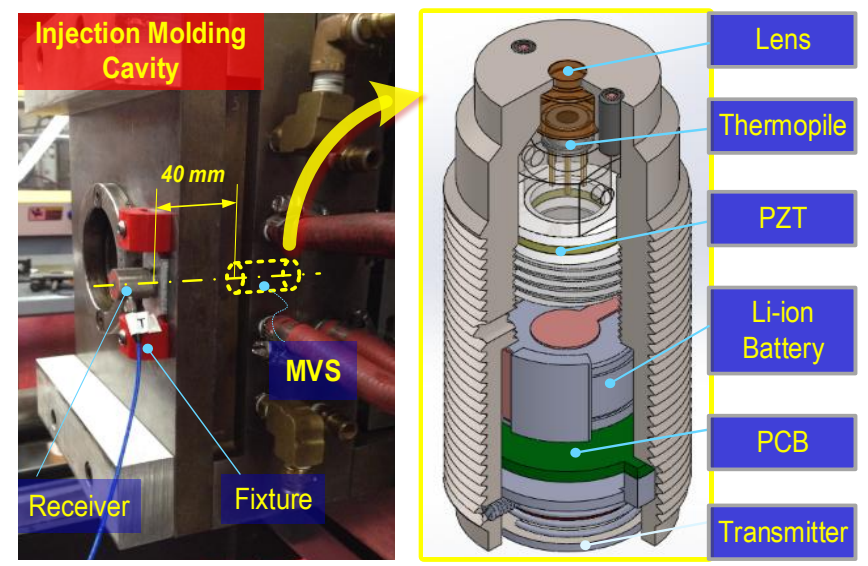

Fig. 11. Experimental setup of injection molding machine.

The sensor package consists of a piezoceramic stack, a thermopile, an ultrasonic transmitter, a steel cylindrical housing, and on-board electronics [15]. The thermopile measures the temperature of the polymer melt as it flows across a zinc selenide ( $\mathrm{ZnSe})$ lens. The pressure from the polymer melt is measured by the piezo-ceramic stack via a steel stand-off that protects the thermopile. The ultrasonic transmitter is located at the bottom of the sensor package, and is coupled with the injection mold steel. The piezoelectric effect converts the melt pressure into a 
proportional electrical charge output and provides a means for harvesting energy from the pressure associated with the injection molding process. This energy is used for powering the sensor electronics (in conjunction with a rechargeable battery, controlled by a hybrid powering algorithm). The measured physical parameters are converted to digital signals, and subsequently modulated by sinusoidal carrier waves of designed frequencies (e.g. $0.8 \mathrm{MHz}$ and $1 \mathrm{MHz}$ ), generated by the on-board electronics. The resulting electrical signal is applied to the piezoelectric layer of the transmitter to convert the signal into an ultrasonic (mechanical) wave, which will then be transmitted to the external ultrasonic receiver. Fig. 12a shows the waves obtained by the external ultrasonic receiver, and the spectrum of the ultrasonic waves is illustrated in Fig. 12b. From the spectrum analysis, the frequencies of the ultrasonic carrier waves at $0.8 \mathrm{MHz}$ and $1 \mathrm{MHz}$ are clearly identified, respectively. The transmitted digital signals, "100011111" for the melt temperature and "010111000" for the melt pressure, could be obtained from the recording of the MVS at the corresponding time. The last digit of each signal is the parity bit used for transmission error checking.
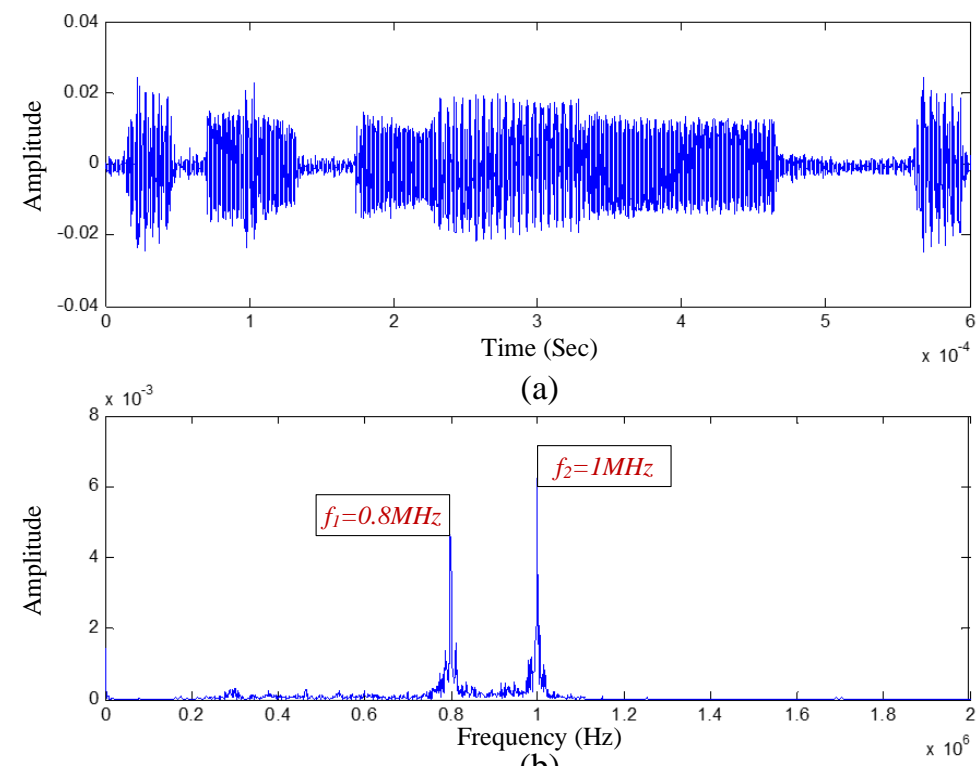

(b)

Fig. 12. Experimental ultrasonic signal and its spectrum. 
Next the ultrasonic signal is fed into DT-CWPT, and the decomposition results of DTCWPT at the fourth level are obtained as shown in Fig. 13. The wavelet coefficients in blue colour are the real part, while the ones in red colour are the imaginary part. According to the criteria maximum relative energy to Renyi entropy ratio, two wavelet coefficients (as highlighted in Fig. 13) are selected. They correspond to the two ultrasonic carrier waves at frequencies 0.8 $\mathrm{MHz}$ and $1 \mathrm{MHz}$, respectively. The envelopes are extracted from the DT-CWPT by combining the real part and imaginary part of the selected wavelet coefficients. The results are shown in Fig. 14. By identifying the head of the data package, the envelopes are then divided into sized sections. The mean value of each section is then calculated and compared with the adaptive threshold as discussed in section 3.4. Comparison between the transmitted digits ("100011111" for melt temperature and " 010111000 " for melt pressure) and the received 8-bit digital data package confirmed that the intended information was correctly transmitted and the retrieved by the ultrasound receiver, as highlighted in Fig. 14. From the above analysis, the effectiveness of the presented MuSEnSA method is demonstrated for signal separation and symbol retrieval.
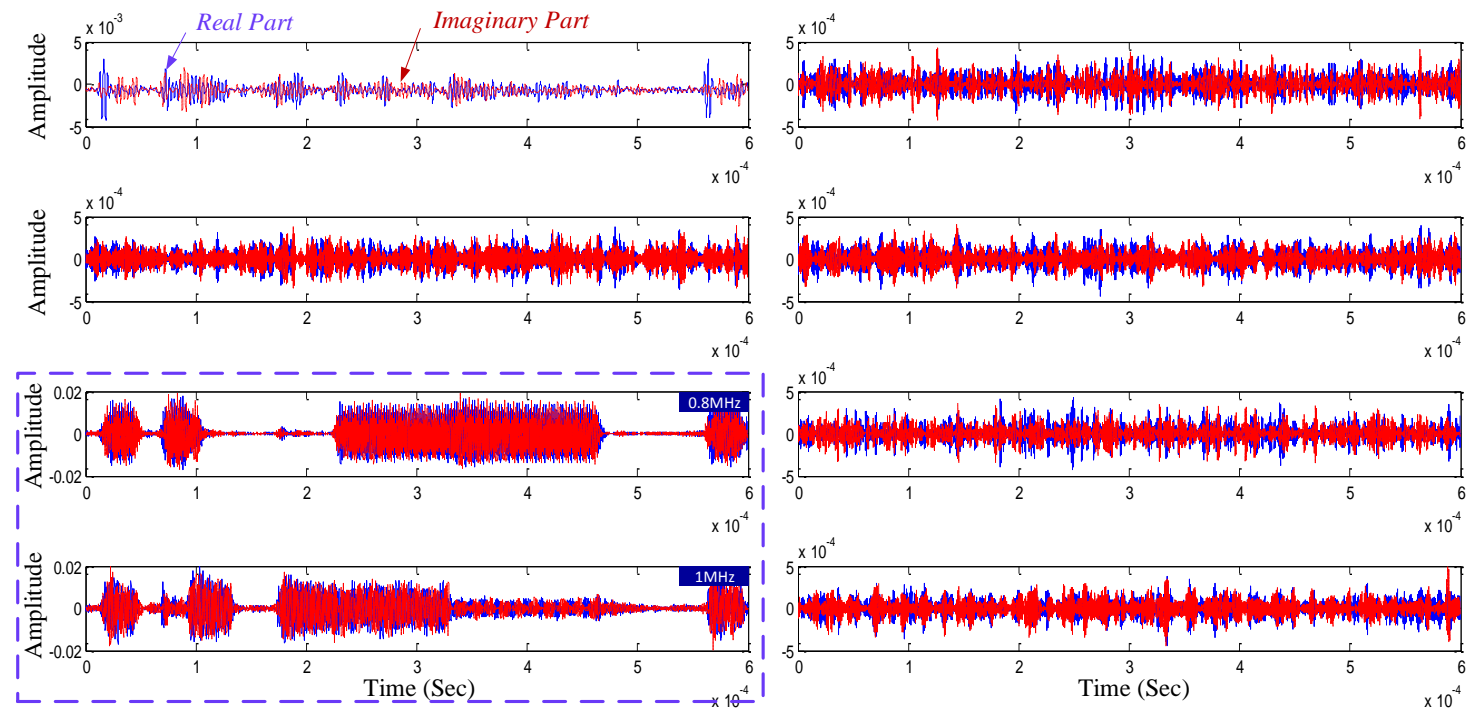

Fig. 13. Part of decomposition results (including real part and imaginary part) of DT-CWPT at the decomposition level 4. 


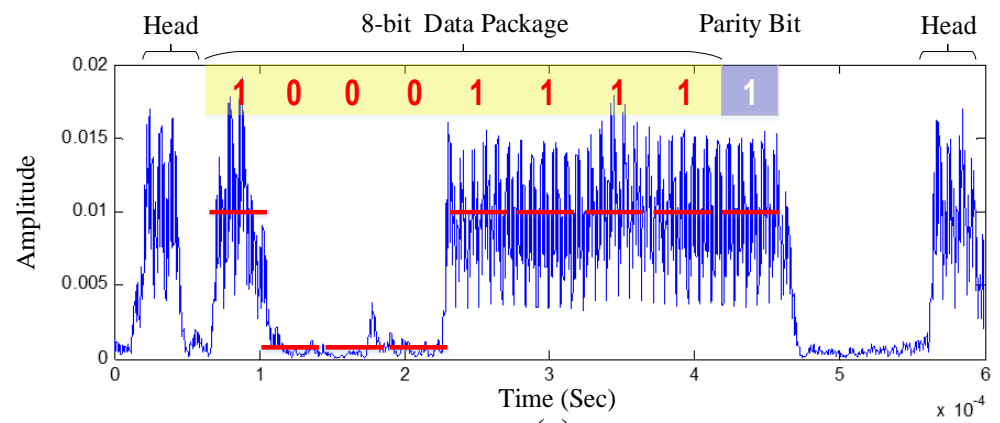

(a)

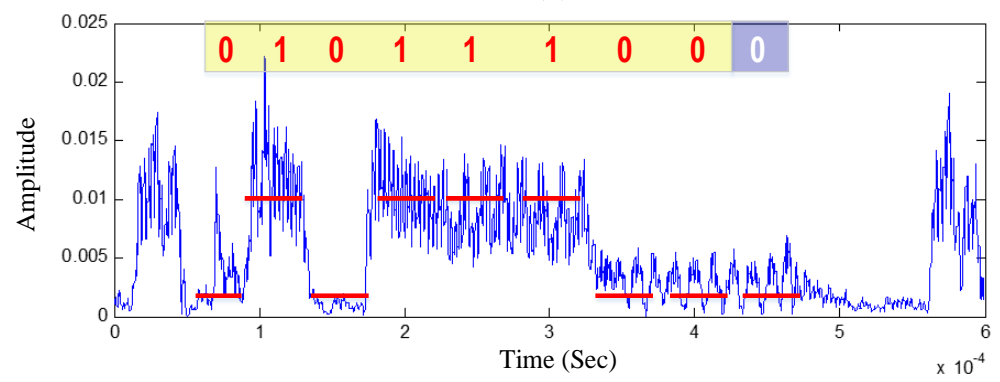

(b)

Fig. 14. The envelopes extracted from selected wavelet coefficients and retrieved digital data using symbolic representation analysis with carrier frequency, a) $0.8 \mathrm{MHz}$, and b) $1 \mathrm{MHz}$.

\section{CONCLUSIONS}

This paper investigates a multivariate ultrasonic signal transmission mechanism through metal structures. On the transmitter side, a multi-carrier amplitude modulation scheme has been developed to increase the ultrasonic data transmission rate. On the receiver side, the MuSEnSA method has been presented to extract the physical information, measured by the in-mold sensor and coded as digital data, from the ultrasonic waveform. Through demodulation, the envelopes of the coded data are successfully obtained from the ultrasonic carrier waves by the DT-CWPT algorithm. Then the coded data is retrieved based on the symbolic representation analysis. The effectiveness of the developed method is experimentally demonstrated in an injection molding machine using a prototype of an embedded multivariate sensor. 
A number of research issues, such as the effect of the transmission data length, offset of the transducer position, signal-to-noise ratios, and material properties, will be investigated in the future research.

\section{ACKNOWLEDGMENTS}

This research is partially supported by the National Science Foundation of America (CMMI1000816), Natural Science Foundation of China (No. 51504274 and No. 51674277), and Science Foundation of China University of Petroleum, Beijing (No. 2462014 YJRC039 and No. 2462015YQ0403). Experimental support from University of Connecticut and University of Massachusetts, Lowell is greatly appreciated. Valuable comments from the anonymous reviewers are greatly appreciated to help improve the quality of the paper.

\section{REFERENCES}

[1] E. Egea-Lopez, A. Martinez-Sala, J. Vales-Alonso, J. Garcia-Haro, J. Malgosa-Sanahuja, Wireless communications deployments in industry: a review of issues, options and technologies, Computers in Industry, 56(2005)29-53.

[2] X. Cheng, A. Datta, H. Choi, X. Zhan, X. Li, Study on embedding and integration of microsensors into metal structures for manufacturing applications, Transactions of ASME, Journal of Manufacturing Science and Engineering, 129(2007) 416-424.

[3] K. Lu, A plug-and-play data gathering system using ZigBee-based sensor network, Computers in Industry, 62(2011)719-728.

[4] L. Zhang, C.B. Theurer, R.X. Gao, D.O. Kazmer, Analytic wavelet-based ultrasonic pulse differentiation for injection mold cavity pressure measurement, Transactions of ASME, Journal of Manufacturing Science and Engineering, 128(2006) 370-374.

[5] C. Li, D.A. Hutchins, R.J. Green, Short-range Ultrasonics digital communications in air, IEEE Transactions on Ultrasoincs, Ferroelectrics, and Frequency Control, 55(4)(2008)908918.

[6] K. Wanuga, M. Bielinski, R. Primerano, M. Kam, K.R. Dandekar, High-data-rate ultrasonic through-metal communication, IEEE Transactions on Ultrasonics, Ferroelectrics, and Frequency Control, 59(9)(2012)2051-2053.

[7] T. Hosman, M. Yeary, J.K. Antonio, Design and Characterization of an MFSK-based transmitter/receiver for ultrasonic communication through metallic structures, IEEE Transactions on Instrumentation and Measurement, 60(12)(2011)3767-3774.

[8] H. Du, J.A. Turner, Ultrasonic attenuation in pearlitic steel, Ultrasonics, 54(2014)882-887. 
[9] R. Gao, Z. Fan, N. Asadizanjani, D. Kazmer, Acoustic-based wireless signal transmission for precision metrology: accuracy and reliability, CIRP Annals-Manufacturing Technology, 62(2013)519-522.

[10] A. Abbate, J. Koay, J. Frankel, S.C. Schroeder, P. Das, Signal detection and noise suppression using a wavelet transform signal processor: application to ultrasonic flaw detection, IEEE Transactions on Ultrasonics, Ferroelectrics, and Frequency Control, 44(1)(1997)14-26.

[11] G. Andria, F. Attivissimo, N. Giaquinto, Digital signal processing techniques for accurate ultrasonic sensor measurement, Measurement, 30(2001)105-114.

[12] V. Matz, R. Smid, S. Starman, M. Kreidl, Signal-to-noise ratio enhancement based on wavelet filtering in ultrasonic testing, Ultrasonics, 49(2009)752-759.

[13] H. Chen, M.J. Zuo, X.Wang, M.R. Hoseini, An adaptive Morlet wavelet filter for time-offlight estimation in ultrasonic damage assessment, Measurement, 43(2010)570-585.

[14] G. Shi, X. Chen, X. Song, F. Qi, A. Ding, Signal matching wavelet for ultrasonic flaw detection in high background noise, IEEE Transactions on Ultrasonics, Ferroelectrics, and Frequency Control, 58(4)(2011)776-787.

[15] Z. Fan, R.X. Gao, N. Asadizanjani, D.O. Kazmer, Acoustic wave-based data transmission for multivariate sensing, IEEE Transactions on Instrumentation and Measurement, 62(11)(2013)3026-3034.

[16] I. Bayram, I.W. Selesnick, On the dual-tree complex wavelet packet and M-band transforms, IEEE Transactions on Signal Processing, 56(6)(2008)2298-2310.

[17] I.W. Selesnick, R.G. Baraniuk, and N.G. Kingsbury, The dual-tree complex wavelet transform, IEEE Signal Processing Magazine, 22 (6)(2005)123-151.

[18] T. Weickert, C. Benjaminsen, U. Kiencke, Analytic wavelet packets - combining the dualtree approach with wavelet packets for signal analysis and filtering, IEEE Transactions on Signal Processing, 57(2)(2009)493-502.

[19] I.W. Selesnick, Hilbert transform pairs of wavelet bases, IEEE Signal Processing Letter, 8(6)(2001)170-173.

[20] J. Wang, R.X. Gao, R. Yan, A hybrid approach to bearing defect diagnosis in rotary machines, CIRP Journal of Manufacturing Science and Technology, 5(4)(2012)357-365.

[21] D. Xu, D. Erdogmuns, Renyi's entropy divergence and their nonparametric estimators, Information theoretic learning, Springer, (2010), pp. 47-102.

[22] E. Keogh, K. Chakrabarti, M. Pazzani, S. Mehrotra, Dimensionality reduction for fast similarity search in large time series databases, Knowledge and Information Systems, 3(3)(2000)263-286. 